\title{
INTEGRATIVE IRT FOR DOCUMENTATION AND INTERPRETATION OF ARCHAEOLOGICAL STRUCTURES
}

\author{
M. Griffo ${ }^{1, *}$, P. Cimadomo ${ }^{2}$, S. Menconero ${ }^{1}$ \\ ${ }^{1}$ Dept. of History, Representation and Restoration of Architecture, Sapienza University of Rome, Piazza Borghese 9, Roma, IT - \\ (marika.griffo, sofia.menconero)@uniroma1.it \\ ${ }^{2}$ Dept. of Humanities, University of Naples Federico II, Via Nuova Marina 33, Napoli, IT-paolo.cimadomo@ unina.it
}

Commission II, WG II/8

KEY WORDS: photogrammetry, infrared thermography, IRT, integration data, cultural heritage, Nymphaeum of Egeria

\begin{abstract}
:
The documentation of built heritage involves tangible and intangible features. Several morphological and metric aspects of architectural structures are acquired throughout a massive data capture system, such as the Terrestrial Laser Scanner (TLS) and the Structure from Motion (SfM) technique. They produce models that give information about the skin of architectural organism. Infrared Thermography (IRT) is one of the techniques used to investigate what is beyond the external layer. This technology is particularly significant in the diagnostics and conservation of the built heritage. In archaeology, the integration of data acquired through different sensors improves the analysis and the interpretation of findings that are incomplete or transformed.

Starting from a topographic and photogrammetric survey, the procedure here proposed aims to combine the bidimensional IRT data together with the 3D point cloud. This system helps to overcome the Field of View (FoV) of each IRT image and provides a threedimensional reading of the thermal behaviour of the object. This approach is based on the geometric constraints of the pair of RGBIR images coming from two different sensors mounted inside a bi-camera commercial device. Knowing the approximate distance between the two sensors, and making the necessary simplifications allowed by the low resolution of the thermal sensor, we projected the colour of the IR images to the RGB point cloud. The procedure was applied is the so-called Nymphaeum of Egeria, an archaeological structure in the Caffarella Park (Rome, Italy), which is currently part of the Appia Antica Regional Park.
\end{abstract}

\section{INTRODUCTION}

The documentation of archaeological and architectural built heritage passes by a series of activities devoted to the collection of heterogeneous data concerning the object itself in its tangible and intangible features. The way these data are managed together and visualized influences the following steps of data analysis and reading of the object. Currently, most of the morphological and metrical aspects are acquired through massive data capture systems such as Terrestrial LaserScanning (TLS) and Structure from Motion (SfM). These systems mainly provide point clouds. Point clouds are numeric representation of the reality, and as such they describe the external skin of the real object with a measurable accuracy. Survey operations are mostly completed with the production of 2D and 3D models representing an interpretation of the spatial and geometrical aspects of this external layer.

Recently, the technological advancing in sensors and IT solutions allows us to test procedures to transform data from different sources to a more organic and integrated model. This is particularly meaningful in the fields related to diagnostics and conservation of the built heritage. As a part of a wider system of information connected to the object as-it-is, non-destructive investigations supply data related to what is under the skin of the object. The spatial association between what is visible on the surface of the object and what is not, becomes crucial for everyone who is involved into the conservation process. In the field of cultural heritage, the integration of data acquired from different sensors improves the analysis and interpretation of archaeological artefacts that are currently incomplete or transformed. It allows to identify the changes occurred over the centuries and to hypothesize the various historical configurations.
In this context, Infrared Thermography (IRT) was used to support the interpretation of the architectural transformations to get a deeper knowledge of the object.

Starting from a SfM photogrammetric and topographic survey, the procedure here proposed aims to combine together IRT twodimensional data with a 3D survey point cloud. This system helps to overcome the limited Field of View (FoV) of each IRT images and to give a three-dimensional understanding of the thermal behaviour of the object.

The thermographic data itself makes the three-dimensional mapping operation coherent with its interpretation. The analysis of the data, in fact, gives information about what happens beyond the structure, in the non-visible part of it, even if the measurement of the data corresponds to the radiation identified on the object surface.

This peculiarity allows, on one hand, to control and manage the image as a thematic mapping of the visible surface and, on the other hand, to understand three-dimensional phenomena starting from "two-dimensional hints". The thermal data gives us an image of the object in an intensity scale. This image is often difficult to trace back to the image that our eyes acquire into the visible spectrum. For this reason, the infrared sensor of the camera is used together with an RGB camera with standard sensor. In this way, the values of thermal intensity relate to their corresponding values in the visible spectrum. The pair constituted by an RGB image and the related IRT image allows spatial operations of roto-translation and projection, that are useful to providing a three-dimensional mapping of the data. The work here presented shows the procedure used for handling IRT images in a three-dimensional virtual space. Furthermore, it is connected with the broader theme of the integration processes between heterogeneous data referred to the cultural heritage.

\footnotetext{
* Corresponding author
} 


\section{RELATED WORKS}

Several studies have been conducted on this topic focusing on various stages of the whole procedure.

Starting from different tests performed to verify the accuracy and calibration of sensors (Hess et al., 2014; Rangel et al., 2014), the focus is to develop strategies for the integration of thermographic data with $3 \mathrm{D}$ point clouds.

If the surface of the object is essentially bidimensional, the problem could be solved projecting single images on a fitting plan, this procedure works on two dimensions and does not consider any $3 \mathrm{D}$ feature of the object, it can be considered as a first level of integration that may be useful at a certain specific condition (Costanzo, 2014).

Experimentations carried on this topic mainly rely on the spatial reconstruction of tie points visible on both RGB and IR images (Scaioni et al., 2017; Clarkson et al., 2017). Another approach is to combine TLS point clouds and 2D images through the extraction of line features from which is possible to compute orientation parameters, standardized as a combination of spatial movements (Lagüela et al. 2013). The integration of 2D thematic images with TLS point cloud can be carried out solving the collinearity equations for image registration by using Ground Control Points (GCPs) to detect correspondent points (Previtali et al., 2013).

The main issue for all those procedures based on feature detection of images are the low resolution of the frame and the gradual variation of temperature registered on the surface of the objects. The gradual variation of temperature affects the sharpness of the image, for this reason, the transition between different types of material or state of conservation is generally smooth. With this premises, the IR image recognition of points and edges can be very challenging.

In this context, a valid alternative is to test a procedure that calculates the camera location of the IR sensor considering spatial and geometrical references with respect to the system of coordinates of the 3D point cloud. Following this line, some valid experimentations are focused on using customized devices with a fixed and known geometry between IR and RGB sensors (Alba et al., 2011), this system, named "bi-camera", allows to reconstruct the exterior orientation (EO) and the relative orientation of the IR camera by imposing a fixed distance and position between the RGB and IR cameras. Following this line, other studies have been conducted using commercial devices with a built-in IR sensor and a standard one. The mutual fixed geometry of the two sensors permits to derive the local coordinates of the IR sensor starting from the external orientation parameters of the standard one (Ham et al.,2013). This solution provides a reproducible workflow and appears to be largely applicable whenever there is a lack of control points on the IR image.

\section{CASE STUDY}

The so-called "Nymphaeum of Egeria" is a well-known structure located in the Caffarella Park, that is currently part of the Appian Way Regional Park and stretches from the Aurelian Walls up to the river Almone shores (Dubbini, 2017, 57).

From the $2^{\text {nd }} / 1^{\text {st }}$ century BCE onwards, part of the area was property of the gens Annia, one of the most important families of Rome. During this period, other Roman families started to build nearby their villae, namely country houses where servants carried out several farming activities for their owners. During the imperial period, in particular in the $2^{\text {nd }}$ century, there was a general renovation and improvement of these houses. The most important villa was still the property of gens Annia. One of the most important owners was Annia Regilla, wife of Herodes Atticus, a Greek nobleman who became Roman Senator and funded several public buildings, especially in Greece. Herodes was an important figure in the imperial circle and was the tutor of Marcus Aurelius and Lucius Verus, who became emperors later. Annia Regilla and Herodes Atticus started the erection of a big villa in their possession. The area they owned was so large that it also included a pagus namely a village inhabited by servants and coloni (tenant farmers). The principal structure of their estate was located on a small hill and sustained by a cryptoporticus with rooms with frescoes. When Anna died, her husband erected a sanctuary, called Triopion, but we have no information about its location (Dubbini, 2017, 60). Among the structures built there, the Nymphaeum constitutes the best preserved one: it was probably a sort of artificial grotto exposed to the southern shore of the river Almone (specus aestivus) (Kammerer-Grothaus and Kocks, 1983; De Cristofaro, 2014, 44). Later on, during the $4^{\text {th }}$ century, the Nymphaeum became part of the estate of Maxentius, who partly modified it connecting an aqueduct to the back side of the monument and adding a basin. In this way, the grotto became a monumental fountain. The Nymphaeum probably preserved its function as fountain in the Middle Age and later, as attested by the several drawings from the $16^{\text {th }}$ century onward, when the Nymphaeum was visited, described and studied by several artists and scholars (De Cristofaro, 2013). It has been traditionally identified as the grotto of Egeria, a nymph loved by Numa Pompilius, the second legendary king of Rome. In $19^{\text {th }}$ century, however, some scholars started to have doubts about the identification of the structure: Carlo Fea, who started scientific excavations between $1820 \mathrm{~s}$ and $1830 \mathrm{~s}$, was the first to propose to correct the mistake, followed by Rodolfo Lanciani at the end of the same century. At the end of 1990s, new archaeological excavations and restorations were resumed, better clarifying the function and the history of the Nymphaeum.

\section{METHODOLOGY AND WORKFLOW}

This contribution is part of a wider research, still in progress, whose focus is to study the hypotheses of reconstruction of the various phases of the Nymphaeum of Egeria and the dissemination of the results through an application of Augmented Reality (AR) on site.

In this first part, the work is focused on the collection of information, preparatory to the formulation of the reconstructive hypotheses. The survey operations of the Nymphaeum were carried out with the aim of obtaining information of a metric, geometric, functional and decorative nature. For this reason, a thermographic campaign accompanied the traditional geometric survey. After the processing phase, the two kind of data were merged to allow the reading of the thermal component directly in the three-dimensional space of the model.

\subsection{Acquisition}

The field work concerned the acquisition of different types of data: high-resolution photographs, used for the geometric reconstruction of the archaeological structure; thermographic images of some portions of the masonry selected on the basis of interest in the reconstructive hypotheses of the ancient building; topographic measurements to obtain indispensable control points used to scale the object and verify the geometric reconstruction.

The topographic campaign was conducted with a Leica TS02 total station. Markers of Agisoft Photoscan, which is the software used for the photogrammetric process, materialized the measured points. The markers were placed in the lowest and 
most articulated part of the Nymphaeum, taking care to place them at different heights. 15 points were collected on the archaeological structure (Fig. 1), in addition to about ten points on the walkway from which you can enjoy the view of the Nymphaeum, which will be used later for the realization of the AR app. A monograph was recorded for each point to make recognition clearer and faster.

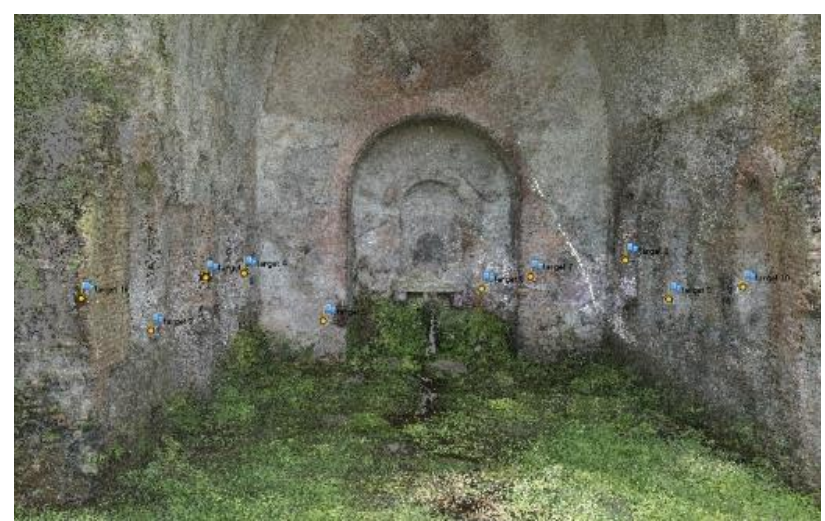

Figure 1. Sparse point cloud with markers location

The main photographic campaign was carried out with a Nikon D800E SLR camera and a $35 \mathrm{~mm}$ lens. The shooting settings are as follow: 200 ISO, F/6.3 aperture and 1/100 shutter. 683 photos were taken from different points of view: both from the Nymphaeum level and from the walkway facing it (Fig. 2).

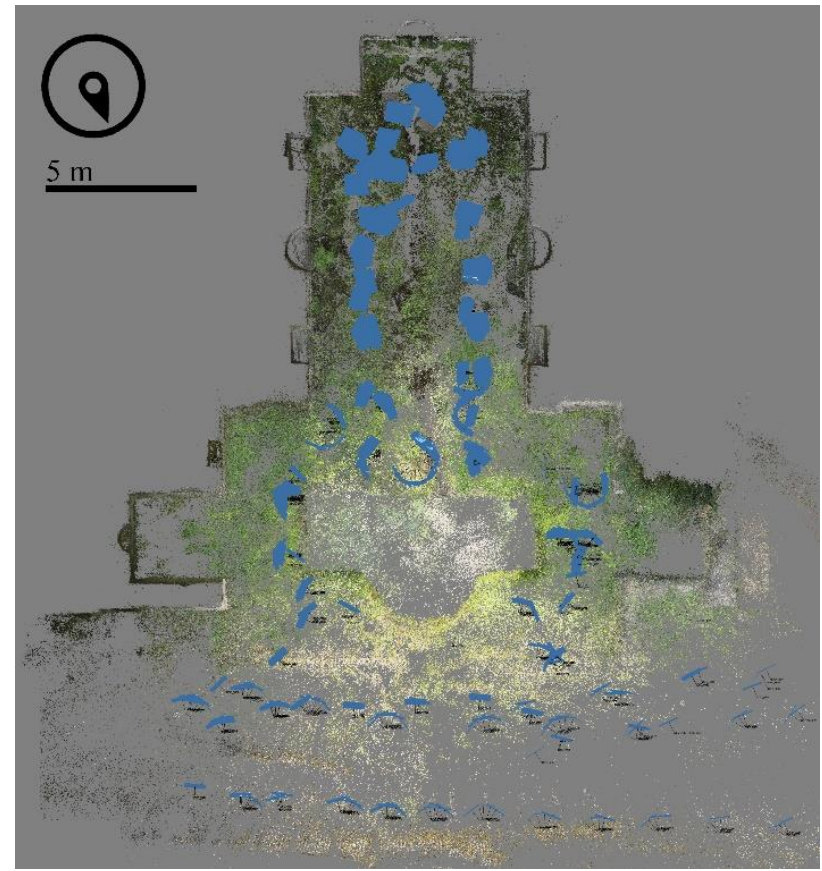

Figure 2. Sparse point cloud with cameras location

Two other photo campaigns were conducted with a Nikon D3300 camera and the integrated camera of a Huawei P10 smartphone. The latter two acquisition were used to compare the results obtained from the processing of each of the three different sensors. The images of the D800E camera were used as a benchmark to assess the impact of the photographic sensor on the metric and geometric reliability of the reconstructed point cloud. The subject will not be explored in this study for reasons of extension.

The Nymphaeum of Egeria, about $12 \times 7$ meters in plan in the area still covered by a barrel vault, is oriented towards the north-east: it is a small cave underneath an artificial hill built specifically for this purpose (Fig. 3).

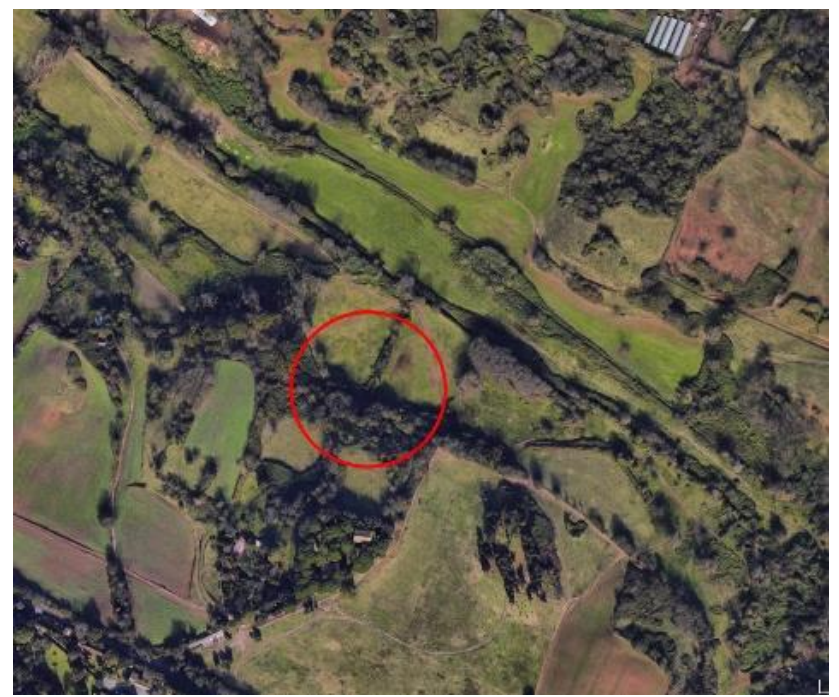

Figure 3. Location of the Nymphaeum in the Caffarella Park

Its orientation, together with the orography of the place, means that the three main sides of the structure are permanently in shadow. Considering these environmental conditions, the thermographic data were acquired passively. In this case, the measurement is carried out only once because the thermal behavior of the structure is not affected by solar radiation. Two campaigns were carried out and, in both cases, a Testo $875 \mathrm{i}$ thermal camera was used, which simultaneously records the thermal and RGB data of the objects photographed.

During the first campaign, the lower band of the south-west wall and, partially, of the south-east and north-west walls was recorded, up to a height equal to half of the niches of the side walls. The thermal camera was positioned at a fixed distance from the walls to keep the Ground Sampling Distance (GSD) constant in all shots. For the same reason, the images were taken as perpendicular as possible to the surface, to avoid excessive distortion of the projected pixel. In the first campaign, the acquisitions were carried out keeping a distance of about 2 meters from the walls of the structure and 56 images were taken. It was also decided to plan a second campaign to investigate in greater detail a clay pipe, partially visible in the north-east wall. 93 images were taken at about $50 \mathrm{~cm}$ from the wall in question. The purpose of the new acquisition was to read the path of the pipe that in the first campaign did not produce results because the thermal difference between the areas of full masonry and those with the pipe inside them was almost zero. In the second campaign we tried to increase the thermal delta by inserting in the pipe another tube filled with very hot water. Unfortunately, the results obtained do not allow a clear reading of the pipe path. The northern exposure of the structure and the constant shading meant that the temperature of the walls was very low even in the summer months and the active heating by means of the tube did not have the expected effect. Other electrical solutions, such as the use of lamps to heat the surfaces, have been excluded for logistical reasons, because the electricity supply is not particularly simple within a park, and 
for safety reasons, because the Nymphaeum floor level is completely flooded by the water of the fountain.

\subsection{Processing}

The processing of the photogrammetric data was done with Agisoft Photoscan Pro, version 1.4.1. The photographs set consisted of 739 images: 683 taken with the Nikon D800E camera and 56 RGB images from the first thermographic acquisition. The thermographic data, which was integrated with the point cloud, is that relating to the first acquisition because it involved a larger masonry sector than the second acquisition. The frames from the SLR camera and the thermal one have very different sizes and resolution. The size of the SLR pictures is $7360 \times 4912$ pixels, while the RGB images of the thermal camera are $640 \times 480$ pixels. To improve the alignment of low-resolution photos, it was decided to process them together with highresolution ones in a single chunk, thus obtaining a single point cloud (Fig. 4). The report data of the aligning process, performed with highest accuracy, indicates an average shot distance of about 6.30 meters and an average ground resolution of $0.95 \mathrm{~mm} / \mathrm{px}$. The identified tie points are 5,528,823 and the reprojection error is below 1 pixel. The total root mean square (RMS) error of the $\mathrm{x}, \mathrm{y}, \mathrm{z}$ coordinates of the control points is 7 $\mathrm{mm}$. The processing time for this phase was 17 hours for matching and 1 hour and 30 minutes for alignment.

The dense cloud (Fig. 5), which has over 435 million points, was processed in high quality with aggressive depth filtering. The processing time was 13 hours and 30 minutes for the generation of depth maps and 2 days for the generation of dense cloud.

The procedure of projection of the thermographic infrared (IR) data on the point cloud is explained in the following paragraph.

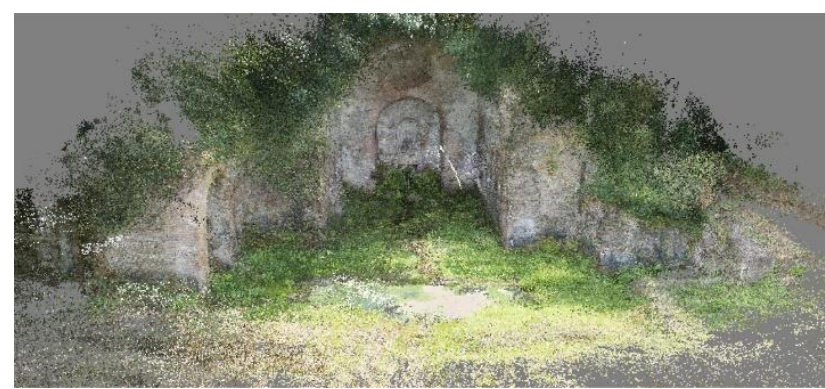

Figure 4. Sparse point cloud of the Nymphaeum of Egeria

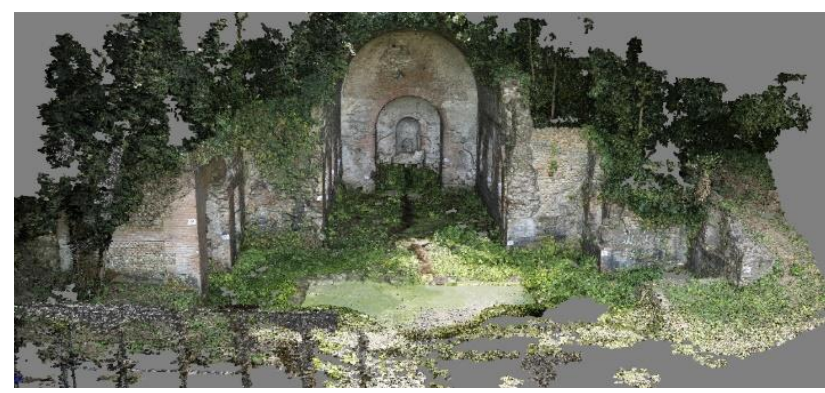

Figure 5. Dense point cloud of the Nymphaeum of Egeria

\section{IRT MAPPING}

The thermal camera used has an RGB and IR dual-sensor system. The two sensors are placed along a vertical axis at a fixed $4 \mathrm{~cm}$ distance. The RGB sensor is located at the bottom and the IR sensor at the top (Fig. 6).

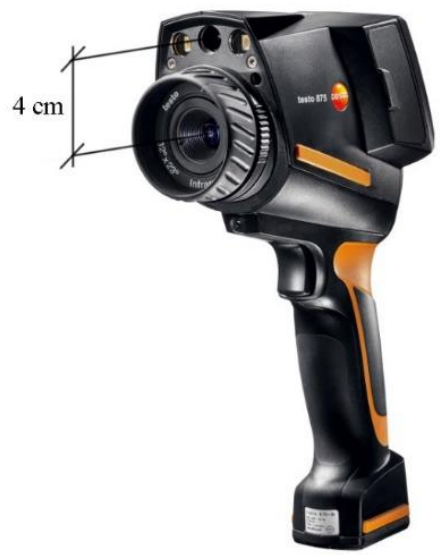

Figure 6. Thermal camera with distance between IR and RGB sensors

The procedure consists of the projection of IRT chromatic component on the point cloud. It uses the geometric constraint that is set up in the pair of RGB-IR images and relies on simplifications allowed by the low resolution of the IR sensor itself (320 x 240 pixels) (1). The standard assumption is based on recording the internal and external orientation of RGB images of the thermal camera, performing a translation of the projection centre on the vertical axis (this translation corresponds to the distance between the sensors), and reprojecting the IR image from the new position to the point cloud. This procedure has been possible following some precautions, for example keeping the thermal camera in a perfectly vertical position during the acquisition phase. This setup has been later verified by superimposing the frames of the pair and verifying that there was only a vertical - and not horizontal - slipping.

The preparatory phase to the alignment operations concerns the two sensors calibration.

First of all, we have corrected the distortion of the two photographic lenses in order to avoid errors during the projection mapping phase. This operation makes the projective system of the cameras uniform and homogeneous over the entire reproduced scene.

RGB images were processed to remove distortion and then exported. This preliminary stage allows to disable the automatic image calibration during the alignment phase in the SfM software.

The related IRT images were exported without distortion directly from the proprietary software of the thermal camera.

The calibration phase is crucial especially for IRT sensors whenever the derived images are used for photogrammetric purposes. This is related to the consistent lens distortion of IR sensors, the low image resolution and the auto-focus function that already makes difficult the parameters estimation (Previtali et al. 2013).

1 The built in IR sensor is $160 \times 120$ pixel but the image exported takes advantage of the SuperResolution option, capable to increase the pixel count by 4 . Each time an image is recorded, a sequence of shoots is saved and then merged together to get a higher-resolution image without any interpolation. 
The RGB images of the pair were processed together with the high-resolution images in order to improve their alignment in the photogrammetric software, as said above.

The average re-projection error is less than one pixel, even considering only the low-res RGB images. Then, the bundle.out file has been exported from Photoscan. The file records the roto-translation matrix that characterise the camera orientation. Together with the boundle.out file, we have exported also the list.txt, that shows the list with the name of raster files.

Both files were later imported in MeshLab, a software useful for analysing and modifying meshes and point clouds, keeping attention to link the IR images (not the RGB images) to the list.txt file. The IR images were obviously scaled with the same dimensions and titled like the RGB images. In this software, it was possible to translate the projection centres along the $\mathrm{z}$ axis, applying the $4 \mathrm{~cm}$ value measured on the infrared device. This operation produced a new camera set in which the calculated projection centres reproduce the position in the space of IRT sensor. The dense cloud of the Nymphaeum of Egeria was also imported. The cloud was coloured with the thermal chromatic component through the projection of raster files from the new projection centre. The workflow ends projecting the thermographic images on the numerical model. (Fig. 7).

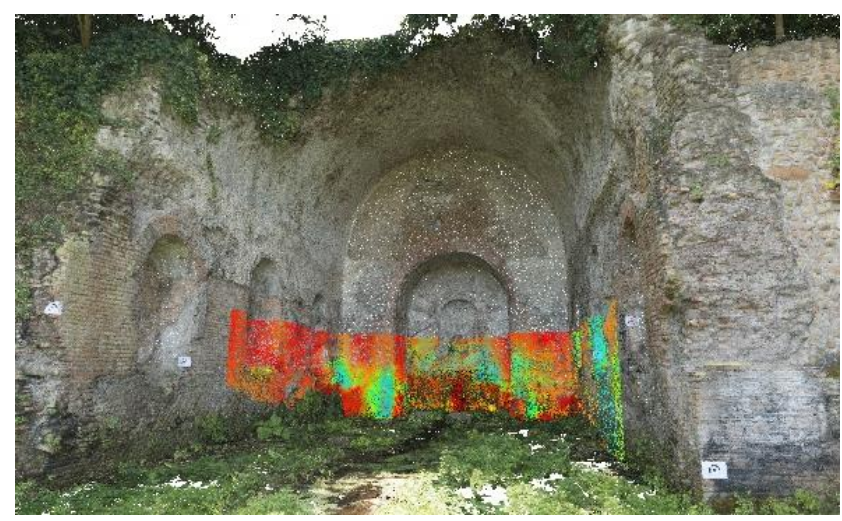

Figure 7. RGB point cloud integrated with IRT data

The examination of metric reliability of the projection centres and of their right positioning occurred by relating the overlapping between the cloud, the RGB image and the thermal image. The operation was carried out on pairs of sample frames with a good recognisability of common characteristic points (Fig. 8, Fig 9). In this way, we had the chance to verify the extension of the translation of the sensor along the $\mathrm{z}$ axis, the correspondence between the two images of the pair and how much reliable was the level of reprojection.

The procedure that we have exposed admits two approximations:

- The real alignment along the $\mathrm{z}$ axis of the two sensors of the thermal camera is hypothesised.

- The translation of the camera took place along the vertical axis of the general reference system. This action does not consider the rolling value of the cameras.

Regarding the first point, the hypothesis of the alignment of the two sensors along the $\mathrm{z}$ axis was proved by verifying that there was no horizontal slip between a pair of RGB-IRT images acquired by keeping the device perfectly vertical.
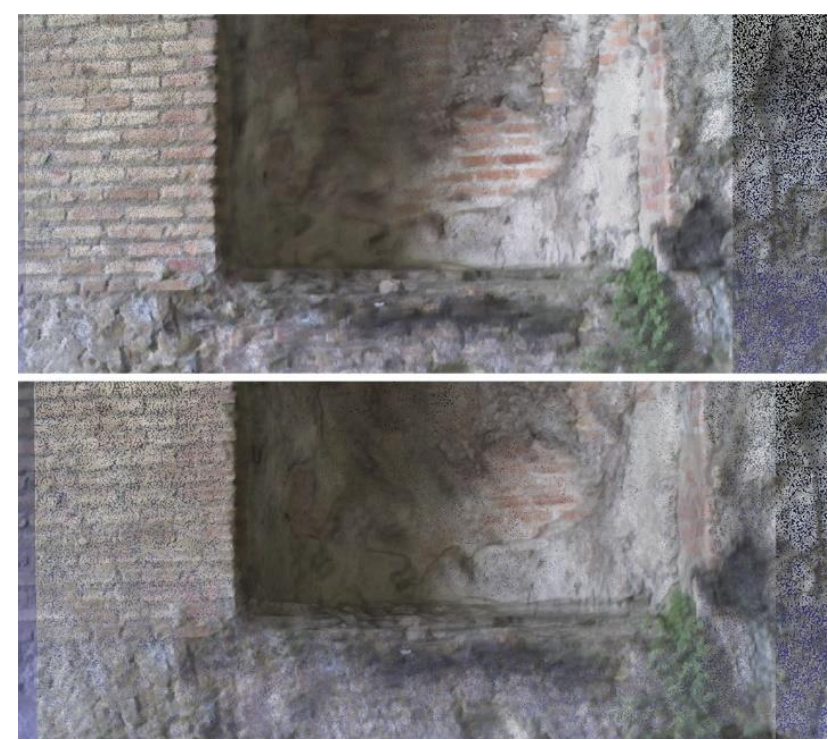

Figure 8. Top: RGB camera rendered on the point cloud in its original position. Down: RGB camera rendered on the point cloud after the $4 \mathrm{~cm}$ down translation
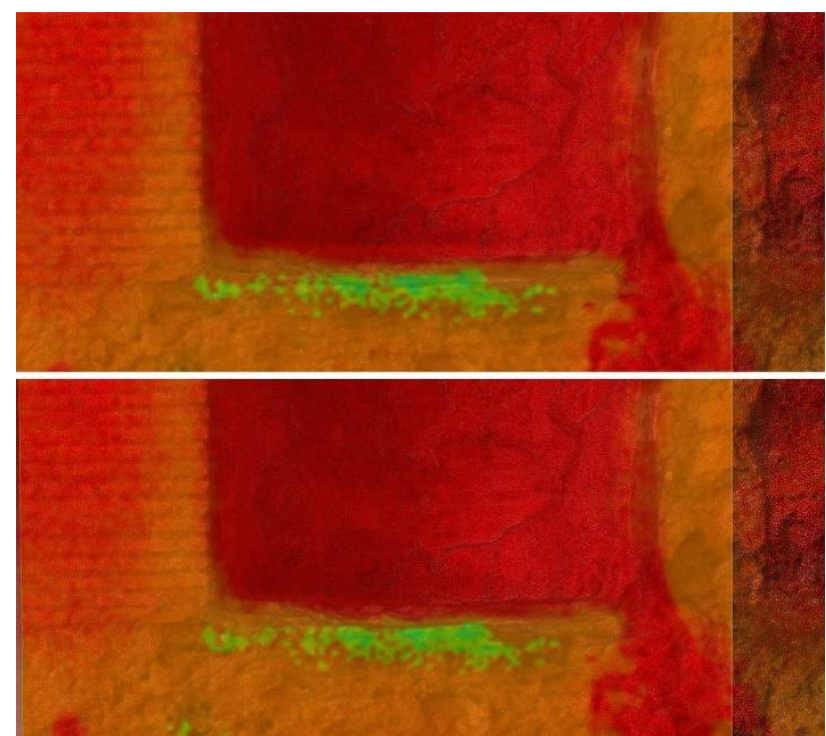

Figure 9. Top: IR camera rendered on the point cloud in its original position. Down: IR camera rendered on the point cloud after the $4 \mathrm{~cm}$ down translation

The second approximation was verified in the acquisition phase avoiding the rolling of the devices. This operation provoked a substantial coincidence between the global $\mathrm{z}$ axis and local $\mathrm{z}$ axis.

During the survey phase, the calculation of the Ground Sample Distance (GSD) of the IR images provided the reference parameter in terms of margin of uncertainty allowed for the mapping operation. The GSD is about $7 \mathrm{~mm} / \mathrm{px}$, with a field angle of $32^{\circ}$, at $2 \mathrm{~m}$ distance (Fig. 10). This approximate value is suitable with the projective operations conducted. 


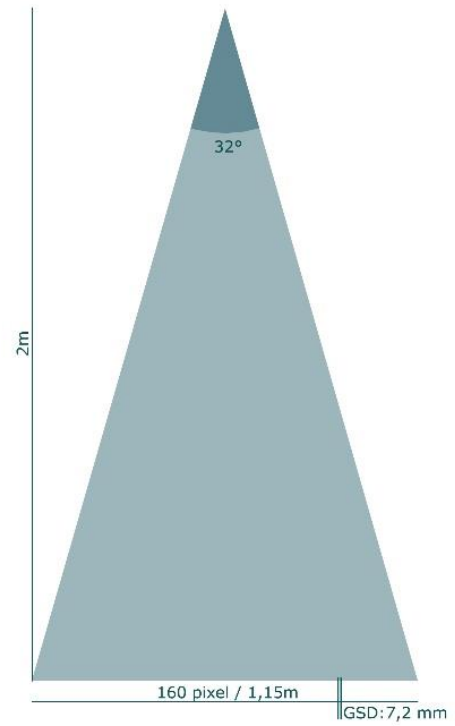

Figure 10. GSD of the IR camera at $2 \mathrm{~m}$ distance from the object

For IR images the GSD parameter by itself cannot provide a complete overview of the image metric and informative control; the auto-focus function cannot be disabled, the colour gradient on the image is smooth, and the Airy disk diameter is larger compared to a standard sensor. Unfortunately, these factors make the GSD not exhaustive to indicate the metric reliability of each shot and therefore this reliability come from a qualitative analysis.

\section{CONCLUSIONS}

The work presented proposes a procedure to integrate IR data with a SfM point cloud. The workflow requires couples of IR and RGB images shot at a fixed and known mutual distance. It leverages on transformations inside the cartesian space and can be applied regardless the IR sensor resolution and the matching of correspondent points between IR and RGB frames. This feature makes it useful whenever the IR data gives back an image with no clear correspondence with the visible spectrum.

The integration of different data typologies into a unique 3D model plays a key role to support research in several fields connected to the cultural heritage. Moreover, a virtual 3D database allows to store data and information and ensures their transmission in an organic way. In this context, the procedure here proposed is just an application of a wider system in which the point cloud becomes the virtual container of all the information connected to the real object. For this reason, we decided to use the point cloud as the final output of the tested procedure, this choice allows to deal with different data coming from manifold sources standardized together as a 3D numeric model.

At the end of the proposed process, the numerical model is featured with the thermographic data, the corresponding point cloud is readable through the intensity value associated with each point.

The temperature value is currently estimated thanks to the colour ascribed to the point, allowing a more qualitative reading. An implementation of the procedure might be the chance of readingthe numerical value of the temperature, connected with the original thermographic data, immediately on the model. It would allow a better interactivity of visualization and interrogation of the thermal points cloud.

The presented research tests at what extent different data can co-exist in the same virtual environment avoiding methodological and procedural contradictions. Once again, the core of this topic is related to the level of transparency that leads the whole process and ensures the repeatability of the procedure (London Charter, 2009).

\section{ACKNOWLEDGEMENTS}

The authors would like to thank the Ente Parco Regionale dell'Appia Antica and expecially Caterina Rossetti and Fabrizio Piccari for their logistics support.

\section{ATTRIBUTIONS}

This contribution is the result of a collaboration between the authors, who agreed to assign themselves the following paragraphs: Case study to P.C.; Introduction, Related works and Conclusions to M.G; Methodology and workflow to S.M. The paragraph IRT mapping has been written by M.G and S.M.

\section{REFERENCES}

Alba, M. I., Barazzetti, L., Scaioni, M., Rosina, E., Previtali, M., 2011. Mapping Infrared Data on Terrestrial Laser Scanning 3D Models of Buildings. Remote Sensing, 3, pp. 1847-1870. https://doi.org/10.3390/rs3091847

Cignoni, P., Callieri, M., Corsini, M., Dellepiane, M., Ganovelli, F., Ranzuglia, G., 2008. MeshLab: an Open-Source Mesh Processing Tool. In: Sixth Eurographics Italian Chapter Conference, pp. 129-136.

Clarkson, G., Luo, S., Fuentes, R., 2017. Thermal 3D Modelling. In: 34th International Symposium on Automation and Robotics in Construction (ISARC 2017), 28 Jun-01 Jul 2017, Taipei, Taiwan, pp. 493-499.

Cho, Y.K., Ham, Y., Golpavar-Fard, M., 2015. 3D as-is building energy modeling and diagnosis: A review of the stateof-the-art, Advanced Engineering Informatics, 29, pp. 184-195.

Costanzo, A., Minasi, M., Casula, G., Musacchio, M, Buongiorno, M.F., 2015. Combined use of terrestrial laser scanning and IRT thermography applied to a historic building. Sensors, 15/2015, pp. 194-217.

De Cristofaro, A., 2002. Valle della Caffarella. Indagini al Ninfeo di Egeria (Municipio XI). In: Bullettino della Commissione archeologica comunale di Roma 103, pp. 233236.

De Cristofaro, A., 2005. Il Ninfeo di Egeria nella valle della Caffarella a Roma: pavimenti e rivestimenti parietali. In: Atti AISCOM X, pp. 613-628.

De Cristofaro, A., 2013. Baldassarre Peruzzi, Carlo V e la ninfa Egeria: il riuso rinascimentale del Ninfeo di Egeria nella valle della Caffarella. In: Horti Hesperidum I, pp. 85-138.

De Cristofaro, A., 2014. Il Ninfeo di Egeria nella valle della Caffarella a Roma. Forma, cronologia, funzione. In: Orizzonti, Rassegna di Archeologia XV, pp. 31-49.

Dubbini, R., 2017. La Valle della Caffarella nei secoli, Roma, Gangemi Editore. 
Ham, Y., Golparvar-Fard M., 2013. An automated vision-based method for rapid 3D energy performance modeling of existing buildings using thermal and digital imagery, Adv. Eng. Inform. 27,2013, pp.395-409.

Hess, M., Kuester, F., Trivedi, M., 2014. Multimodal registration of high-resolution thermal image mosaics for the non-destructive evaluation of structures. In: 2014 IEEE International Conference on Imaging Systems and Techniques (IST) Proceedings, 14-17 october 2014, Santorini, Greece, pp. 216-221.

Kammerer-Grothaus, H., Kocks, D., 1983. Spelonca di Egeria, In: Mededelingen van het Nederlands Instituut te Rome 44, pp. 61-77.

London Charter, 2009. Retrieved from: http://www.londoncharter.org/

Lagüela, S., Díaz-Vilariño, L., Martínez, J., Armesto, J., 2013. Automatic thermographic and RGB texture of as-built BIM for energy rehabilitation purposes. Automation in Construction, $31 / 2013$, pp. 230-240.

Lin, Jarzabek-Rychard, D. Schneider, Maas, 2018. Thermal texture selection and correction for building facade inspection based on thermal radiant characteristics, Int. Arch. Photogramm. Remote Sens. Spatial Inf. Sci., vol. XLII-2, pp. 585-591, 2018, doi: 10.5194/isprs-archives-XLII-2-585-2018.

M. Previtali, L. Barazzetti, V. Redaelli, M. Scaioni, and E. Rosina, 2013. Rigorous procedure for mapping thermal infrared images on threedimensional models of building façades, Journal of Applied Remote Sensing, vol. 7, no. 073503-1, 2013.

Rangel, J., Soldan, S., 2014. 3D Thermal Imaging: Fusion of Thermography and Depth Cameras. In: 12th International Conference on Quantitative Infrared Thermography, 7-11 July 2014, Bordeaux, France.

Ranzuglia, G., Callieri, M., Dellepiane, M., Cignoni, P., Scopigno, R., 2013. MeshLab as a complete tool for the integration of photos and color with high resolution 3D geometry data. In: CAA 2012 Conference Proceedings, pp. 406416.

Sfarra, S., Marcucci, E., Ambrosini, D., Paoletti, D., 2016. Infrared exploration of the architectural heritage: from passive infrared thermography to hybrid infrared thermagraphy (HIRT) approach. In: Materiales de Construcción, Vol. 66, Issue 323, July-September 2016. http://dx.doi.org/10.3989/mc.2016.07415

Scaioni, M., Rosina, E., L'erario, A., Dìaz-Vilariño, L., 2017. Integration of infrared thermography and photogrammetric surveying of built landscape. In: International Archives of the Photogrammetry, Remote Sensing and Spatial Information Sciences, Volume XLII-5/W1, GEOMATICS \&

RESTORATION - Conservation of Cultural Heritage in the Digital Era, 22-24 May 2017, Florence, Italy, pp. 153-160. https://doi.org/10.5194/isprs-archives-XLII-5-W1-1-2017 